\title{
La política exterior de Estados Unidos en Asia a la deriva: de Barack Obama a Donald Trump
}

\author{
Yusuke Murakami*
}

\section{RESUMEN}

Este artículo analiza la política exterior de Estados Unidos en Asia. En dicha región, luego de caer el tradicional sistema vertical y jerárquico de Estados en el siglo XIX y pasar por vaivenes de conflictos y guerras en la primera mitad del siglo XX, la presencia de Estados Unidos permitió el mantenimiento de un statu quo (aunque ciertamente frágil). Este estudio se enfocará, principalmente, en la administración de Barack Obama que introdujo un cambio significativo en relación a la política de Estados Unidos en Asia, así como en la actual administración de Donald Trump la cual presenta una tendencia de retorno al aislacionismo de Estados Unidos.

En efecto, en el siglo XX, la superpotencia desarrolló una política diplomático-estratégica de compromiso con el «orden internacional liberal» basada en la democracia liberal representativa y en la economía de libre mercado, para así evitar la aparición de otro país hegemónico en Europa Occidental o en Asia. Dicha política se basaba en el consenso bipartidario entre los agentes políticos de Washington que daba mayor importancia a Europa Occidental. La administración de Obama cambió esta línea diplomático-estratégica, y dio prioridad a Asia, pero su optimismo e idealismo con respecto a la posición de China, junto con otras consideraciones diplomático-económicas, le permitieron al gigante asiático tomar una serie de acciones que provocaron el cambio del statu quo territorial en Asia. Al final, la nueva línea diplomático-estratégica de Obama no se arraigó en Estados Unidos, y con la llegada al poder de Trump, la política estadunidense en Asia se ha vuelto más confusa y contradictoria. Aunque ha quedado claro que a Trump no le interesa mantener y promover el «orden internacional liberal», todavía no se sabe exactamente qué dirección o rumbo va a tomar su administración con relación a la agenda político-estratégica de las relaciones con Asia. De Obama a Trump, la política de la superpotencia en Asia ha quedado a la deriva.

Palabras clave: política exterior de Estados Unidos, orden internacional liberal, aislacionismo, sistemas de Estados, Asia, China, Corea del Norte.

* Doctor en Ciencia Política y profesor titular de la Universidad de Kioto, Japón. Correo-e: ymurakam@cseas. kyoto-u.ac.jp 
United States' Foreign Policy in Asia Going Adrift: From Barak Obama to Donald Trump SUMMARY

This article analyzes the foreign policy of the United States in Asia, where a fragile status quo with the presence of the United States was established, after falling a traditional vertical and hierarchical States system in the nineteenth century and going through the vagaries of conflicts and wars in the first half of the twentieth century. The main focus will be given to the administration of Barak Obama that introduced a major change on the issue, as well as the current administration of Donald Trump with the tendency to return to the isolationism of the United States. In the twentieth century the superpower had a diplomatic-strategic policy of committing itself to the «liberal international order» based on liberal representative democracy and the free market economy, and avoiding the emergence of a hegemonic country in Western Europe and Asia. This policy was based on the bipartisan consensus among the political decision-makers of Washington, D.C. that gave greater importance to Western Europe. The Obama administration changed this diplomatic-strategic line, and gave priority to Asia, while its optimism and idealism regarding China's position, along with other diplomatic-economic considerations, allowed the giant of Asia to take a series of acts that caused the change to the territorial status quo. The new diplomatic-strategic line of Obama did not take root in the United States in the end, and with Trump's coming to power, United States' policy in Asia has become more confused and contradictory. Although it has become clear that Trump is not interested in maintaining and promoting the «liberal international order", it is still not known exactly what direction or line the Trump administration will take in relation to the political-strategic agenda of relations with Asia. From Obama to Trump, the policy of the superpower in Asia has been going adrift.

Keywords: United States' foreign policy, liberal international order, isolationism, States systems, Asia, China, North Korea

\section{Introducción: la incógnita política de Trump, la caída hegemónica de Estados Unidos y el statu quo frágil en Asia}

Como en el caso de América Latina y otras partes del mundo, la política exterior de Donald Trump en Asia sigue siendo incógnita, confusa, y aparentemente sin brújula ni norte claro, luego de pasar más de un año de su asunción al poder. Ello se debe, primordialmente, a su carácter "populista», al hecho de ser un outsider sin experiencias anteriores en ningún cargo público, un presidente «accidental» elegido como resultado de una serie de circunstancias internas en Estados Unidos, y cuyo estilo es tomar decisiones según la coyuntura, pensando que este modo de hacer política — «flexible» de acuerdo con las circunstancias— es adecuado para su país, al cual da prioridad número uno: «Primero Estados Unidos» (Levitsky y Ziblatt, 2018; Mecanic, 2017). 
Pero para analizar la política exterior de Trump en Asia, hay que entender su estilo político y tomar en cuenta hasta qué grado o hasta dónde la administración de Trump — que simboliza el retorno de Estados Unidos al aislacionismo— retrocede o reduce su alcance o espacio de acción estratégico-diplomática. Por las amargas experiencias de las dos guerras mundiales desarrolladas durante la primera mitad del siglo XX, Estados Unidos abandonó la política del aislacionismo del siglo XIX y asumió el rol de proteger y promover a escala mundial el llamado «orden internacional liberal», basado tanto en la democracia liberal representativa como en la economía abierta de libre mercado. Fiel a su ideal del mencionado orden, Estados Unidos intentó evitar en Asia la aparición de un país hegemónico ajeno al principio de la libertad en la segunda mitad del siglo pasado, manteniendo su presencia en el mundo y en particular en dicha región sobre la base de una serie de alianzas político-militares.

\subsection{Cambio de correlaciones de poder en el mundo y Asia}

Sin embargo, en este siglo, la posición de Estados Unidos en las correlaciones de poder en el mundo ha cambiado. La hegemonía de dicho país, como única superpotencia mundial luego del fin de la Guerra Fría ha caído, en gran medida, debido a la emergencia de la China comunista, que aspira a convertirse en una superpotencia mundial. China es la nación del «capitalismo de Estado», un modelo que, sin eliminar el principio del control estatal de la economía, ha adoptado varios elementos capitalistas, y al mismo tiempo, tiene un régimen autoritario que restringe la libre participación de los ciudadanos en la política. Vale decir que China ha logrado manejar el Estado y acumular el poder suficiente como para competir con Estados Unidos por la hegemonía mundial, rechazando los principios de la democracia liberal representativa y la economía de libre mercado (Murakami, 2017, pp. 11-15).

Lo que agravó la caída de la hegemonía estadounidense fue la depresión y el estancamiento económicos después de la crisis de 2008 , así como la creciente desigualdad socioeconómica como consecuencia de la línea económica neoliberal aplicada en la década de 1980. A diferencia de América Latina, y al igual que los países europeos, Estados Unidos ha experimentado la aparición gradual de los efectos de la política neoliberal por más de veinte años debido a la lenta desintegración del régimen de bienestar establecido después de la Segunda Guerra Mundial ${ }^{1}$. Este cambio socioeconómico interno ha constituido un ambiente favorable para el retorno al aislacionismo (Murakami, 2013; 2017, pp. 15-23).

\footnotetext{
1 En América Latina, el modelo de desarrollo socioeconómico perseguido desde la década de 1930 cayó simultánea y uniformemente en casi todos los países durante la década de 1970; por ello, se vieron obligados a introducir la línea neoliberal rápidamente para la estabilización y recuperación económica. La aparición de sus efectos, positivos y negativos, fue relativamente rápida (entre la década de 1990 y la primera década del siglo XXI).
} 


\subsection{Sistemas de Estados en contraste entre Europa y Asia}

Las preguntas relacionadas con el retorno al aislacionismo de Estados Unidos revisten una importancia crucial para Asia — particularmente para Asia del Este y Japón-, porque el statu quo regional después de la Segunda Guerra Mundial dependió exclusivamente de la presencia de la superpotencia. A diferencia de Europa de los últimos siglos, Asia nunca ha tenido la experiencia histórica de institucionalizar un sistema horizontal de Estados ${ }^{2}$. En Europa, la firma de la Paz de Westfalia en 1648 dio fin a la feroz guerra de los Treinta Años (1618-1648) y, al mismo tiempo, dio origen a un nuevo principio de Estado con soberanía. El principio de soberanía estatal cambió las relaciones entre los Estados del sistema vertical de la época medieval al sistema horizontal basado en la igualdad de trato y de derechos. El sistema europeo de Estados se fortaleció con el paso del tiempo gracias al desarrollo de los Estados europeos.

Lo que debemos tener presente es que este sistema europeo de Estados se institucionalizó en un espacio específico, regido por una especie de «normas interiores de la comunidad familiar de Europa». Su base fue el jus publicum europeum (ley pública europea), normas de los miembros de la comunidad cristiana de larga época medieval. En comparación con el orden interno de un Estado donde existe la autoridad política suprema y centralizada, el orden horizontal europeo de Estados soberanos — llamado también régimen o sistema de Westfalia - fue esencialmente un sistema anárquico donde era normal el «estado de guerra», como señalaba Jean-Jacques Rousseau.

No obstante, el sistema europeo de Estados garantizó mínimamente el orden regional y evitó otra guerra de gran envergadura por un siglo, entre 1814 y 1914: es decir, desde la celebración del Congreso de Viena y la consecuente recuperación del orden europeo destruido por la Revolución francesa y las guerras napoleónicas, hasta el estallido de la Primera Guerra Mundial. Ello fue posible debido al control mutuo y autolimitación de cada Estado europeo producto del equilibrio de poder, a las normas internas imperantes en dicho sistema, así como a la noción de "guerra justa» que manejaban. En aquel entonces, una guerra era considerada «justa» cuando esta se producía de acuerdo con ciertos procedimientos formales compartidos por los Estados-nación europeos. Tal «institucionalización de la guerra», junto con la instalación de zonas de amortiguación y de influencia, el mantenimiento del secreto de las negociaciones diplomáticas y la diplomacia libre de la opinión pública de cada país, fueron las condiciones que permitieron superar las luchas sangrientas — como

\footnotetext{
2 La descripción sobre el contraste de los sistemas de Estados entre Europa y Asia se basa en Murakami (2012, pp. 22-26). Véase el mismo artículo para más detalles. Respecto del contraste de los sistemas de Estado entre Europa y Asia, Kissinger (2014) analiza el tema desde un ángulo similar al nuestro. Sobre la situación de las últimas décadas, véase también Goh (2015).
} 
la guerra religiosa o guerra civil— en los años anteriores y, al mismo tiempo, garantizar la estabilidad y la paz de largo plazo. Sin embargo, estas condiciones se fueron perdiendo gradualmente en la segunda mitad del siglo XIX debido a la emergencia de la democracia de las masas, el dogmatismo del nacionalismo, el avance rápido de la tecnología militar, entre otras razones.

En cambio, Asia nunca tuvo un sistema horizontal de Estados comparable a los países europeos en los siglos XVIII y XIX. Más bien, por más de dos mil años hasta el siglo XIX, esta zona se caracterizó por el sistema vertical y jerárquico de Estados, en el que predominaba la civilización clásica de China. Este mundo asiático tuvo como orden legítimo un sistema unipolar de relaciones verticales y jerárquicas entre Beijing, centro de la civilización, y los "países tributarios» como Estados vasallos y las zonas de los «bárbaros» que se extendieron alrededor de la zona central del Imperio. El sistema de tributos - llamado «orden mundial de China» — fue concebido como círculos concéntricos cuyo centro fue siempre la capital de China, donde se hallaba el emperador.

El mundo de la civilización china se mantuvo aislado de la política europea por mucho tiempo. Luego, fue destruido gradualmente, mientras las potencias europeas expandieron su presencia e influencia en el mundo. Recién en la segunda mitad del siglo XIX, China y Japón fueron incorporados al sistema de tratados y formaron un subsistema del sistema europeo de Estados. En dichos años, el equilibrio de poder en Asia fue, de una u otra manera, posible sobre la base del delicado equilibrio entre el grupo de los países marítimos - Gran Bretaña, Francia, los Países Bajos, Alemania, etc.- y la única potencia en el continente asiático, Rusia. Luego de las disputas entre las potencias imperialistas de Europa, la China semicolonizada y las penínsulas y los archipiélagos alrededor de ella —las zonas de amortiguación como Arabia, India, Malasia, Indochina, Indonesia, las Filipinas, Corea, etc.- se convirtieron en las zonas periféricas (las colonias) de los centros de Europa en el mapa del orden mundial.

Más tarde, Estados Unidos y Japón participaron de la competencia imperialista en esta zona y se requirió establecer un nuevo equilibrio de poder. Pero la situación no solo no se estabilizó por más de diez años, sino que condujo a la guerra del Pacífico entre Estados Unidos y Japón (1941-1945), vinculada estrechamente con la Segunda Guerra Mundial en Europa. Al terminar esta guerra, el orden de Asia se encontró en manos de tres potencias: Estados Unidos, la Unión Soviética y la China revolucionaria. La última se consolidó en 1949 cuando obtuvo la victoria sobre los nacionalistas, quienes fueron arrinconados en Taiwán. Con el paso de tiempo, la correlación del poder entre las tres potencias marcó las zonas de influencia en Asia. Debido a la tensión entre las tres, incrementada por la Guerra Fría en Europa, la inestabilidad regional tuvo un carácter constante en la zona. La Guerra Fría en Europa se convirtió 
en una "guerra caliente» en Asia-Pacífico con las guerras de Corea (1950-1953) y Vietnam (1964-1975). Estas constituyeron la guerra subsidiaria entre las superpotencias. Respecto de la guerra de Corea, las partes llegaron al cese de hostilidades, pero hasta ahora no se ha firmado un tratado de paz, por lo que se encuentran formalmente en estado de guerra. En estos años, entonces, el statu quo fue posible gracias a la presencia geopolítica de Estados Unidos en esta parte del mundo.

Precisamente, en el siguiente punto, analizamos más detalladamente la política exterior de Estados Unidos después de la Segunda Guerra Mundial en Asia. Sin embargo, nos enfocaremos en la administración de Barak Obama (2011-2017), la cual, en nuestro concepto, dio mayor importancia a Asia que a Europa. Luego veremos la incertidumbre de la actual administración de Trump frente al Asia.

\section{Política de Estados Unidos en Asia en el siglo XX y su cambio en la administración de Obama}

\subsection{Consenso de Washington sobre su política en Asia}

Como hemos señalado, las experiencias de las dos guerras mundiales en la primera mitad del siglo pasado provocaron que Estados Unidos abandonara su política tradicional de aislacionismo y liderara el «orden internacional liberal». Sobre la base de dicha convicción — que constituyó un consenso bipartidario en Washington- la superpotencia pretendió evitar la aparición de otro país hegemónico tanto en Europa como en Asia. Al mismo tiempo, la otra política tradicional de "puertas abiertas» — proclamada por Estados Unidos a fines del siglo XX frente a las potencias imperialistas europeas para que permitieran a todos los países sin excepción un acceso libre al mercado de Chinacondujo a la visión geopolítica y económica de conseguir y mantener un acceso libre a Europa y Asia con un mayor potencial económico, así como al Medio Oriente rico en recursos petroleros: otro eje de importancia en la estrategia mundial de Estados Unidos. Para poner en práctica dicha estrategia, la superpotencia dio un gran giro en su política, y construyó un sistema de alianzas político-militares con los países de mayor importancia estratégica. También fortaleció las relaciones económicas con tales países ${ }^{3}$.

Estados Unidos estableció así bases militares en Europa Occidental y Asia Noreste para dejar estacionadas sus tropas, e instaló las bases de abastecimiento en diferentes puntos a lo largo de Europa, Medio Oriente y Asia. También consiguió el consentimiento de los países para el libre tránsito aéreo de las fuerzas armadas estadounidenses. De este modo, Estados Unidos conformó un «frente estratégico» a lo largo de la peri-

\footnotetext{
3 Sobre la política diplomático-estratégica de Estados Unidos en el siglo XX, véase Gaddis (1987, 2005a, 2005b), Kissinger (1994), Leffler (2005), entre otros.
} 
feria del continente euroasiático. Debido al cambio de las armas principales — de los bombarderos estratégicos y bombas atómicas a los proyectiles estratégicos-, la importancia de dicho «frente» bajó; sin embargo, los agentes políticos en Washington continuaron dando importancia a la red de alianzas y las bases militares en diversas partes del mundo como «bienes estratégicos» para mantener y aumentar los intereses geopolíticos y económicos globales de Estados Unidos.

Durante la Guerra Fría, para Estados Unidos, Europa Occidental constituyó el lugar de mayor importancia y valor desde el punto de vista geopolítico y económico. En aquellos años, Europa Occidental demostró su fuerte presencia en los aspectos militares e industriales, y también constituyó el frente principal de la Guerra Fría frente a la desaparecida Unión Soviética y el bloque que este lideraba. Cuando las circunstancias lo requerían - como en la guerra de Corea y la de Vietnam-Estados Unidos intervino con su enorme poderío militar en Asia, pero luego de poner fin a los conflictos, Estados Unidos «retornó» a Europa Occidental, reafirmando su mayor importancia estratégica.

Asimismo, con motivo de las fatigas causadas por algunas guerras, el Congreso de Estados Unidos propuso, en muchas oportunidades, que los países aliados asumieran parte de la responsabilidad de la seguridad regional, obligándoles a fortalecer sus propias fuerzas armadas. Sin embargo, los formuladores políticos en Washington se mantuvieron sin cambiar la visión del sistema de alianzas—cuyo núcleo fue la Organización del Tratado del Atlántico del Norte-OTAN_construido a lo largo de la periferia del continente euroasiático. Para Estados Unidos, Europa y Asia fueron importantes, pero la prioridad en la segunda mitad del siglo XX se encontraba en el viejo continente.

De todos modos, como recuerda un documento interno del Gobierno estadounidense en 1995: «[...] los intereses de Estados Unidos en Asia han sido notablemente consistentes en los últimos dos siglos: paz y seguridad; acceso comercial a la región; libertad de navegación; y la prevención de la aparición de alguna potencia o coalición hegemónica» (U.S. Departament of Defense, 1995) .

\subsection{Giro en la línea diplomático-estratégica}

Después del fin de la Guerra fría, Estados Unidos no ejecutó una operación militar masiva con el envío de tropas al extranjero comparables a la guerra de Corea o la de Vietnam, con la excepción del envío de sus tropas como núcleo de las fuerzas multinacionales en la guerra del Golfo en 1991. Pero con motivo de los atentados del

\footnotetext{
4 La traducción es nuestra. Este documento también se denomina el «reporte de Nye» debido al funcionario responsable en su elaboración. Se refiere a Joseph Nye, destacado profesor de relaciones internacionales de la Universidad de Harvard, y en aquel entonces, secretario asistente de Defensa para Asuntos de Seguridad Internacional.
} 
11 de septiembre de 2001, la administración de George W. Bush (2001-2009) llevó a cabo una intervención militar en Afganistán e Iraq por largo tiempo. Su sucesor Barack Obama tomó la posta en medio de sentimientos antibélicos extendidos en la sociedad estadounidense, y decidió realizar un cambio drástico de política para poner fin a dicha ocupación militar. A diferencia de sus antecesores, Obama no mantuvo como "prioridad a Europa», sino que la cambió hacia la región Asia Pacífico y hasta la definió como la de mayor importancia para Estados Unidos. Ello implicó un cambio en la evaluación geopolítica y económica de la superpotencia. Obama enfatizó que para el futuro de su país la paz y prosperidad en Asia Pacífico era de vital importancia y propuso la intervención estratégica estadounidense de largo tiempo en la región para que la dinámica económica de la misma continuara encarrilándose. Anunciada en noviembre de 2011, esta nueva política estadounidense hacia el Asia primero se llamó pivot (giro) y luego rebalance (rebalanceo)5.

La anterior administración de George W. Bush decidió y ejecutó la intervención militar-estratégica estadounidense de mayor envergadura en Asia. Obama, por su parte, además de mantener la línea principal de la administración anterior, la desarrolló con la nueva denominación para que incluyera múltiples aspectos y tuviera un alcance regional más amplio. Particularmente atribuyó gran valor a la intervención en los países del Sudeste Asiático.

La administración Obama se propuso esta nueva política en Asia básicamente debido a tres factores.

i) Económico. El Asia Pacífico había logrado un dramático desarrollo económico, y Estados Unidos buscó engancharse con la dinámica económica de toda la región para su propio desarrollo.

ii) Geopolítico. En Asia Pacífico, el cambio de correlaciones de poder entre los países se había dado aceleradamente con la pujante emergencia de China, y se había presentado un riesgo cada vez más creciente de que se intensificaran los conflictos entre ellos y eventualmente se perdiera la estabilidad y la paz regional.

iii) Por último, Obama pretendió contribuir a la construcción de un orden regional sobre la base de su «orden internacional liberal». En Asia Pacífico, existen sobrepuestas diversas organizaciones multilaterales tales como el Foro de Cooperación Económica Asia Pacífico (APEC por sus siglas en inglés), la

\footnotetext{
5 Sobre la nueva política de "giro" o "rebalanceo» de Obama, véase Anderson y Cha (2017), Bader (2012), Campbell (2016), Chollet (2016), Clinton (2011, 2014), Silove (2016), entre otras. Bader fue el director senior para Asuntos Asiáticos en el Consejo de Seguridad Nacional del primer mandato de Obama (2009-2011). Campbell fue el secretario asistente de Estado para Asuntos de Asia y Pacífico en la administración Obama (2009-2013). Chollet fue el subdirector principal del consejo de Planificación de Políticas del Departamento de Estado (2009-2011), director senior para Planificación Estratégica del Consejo de Seguridad Nacional (2011-2012), y secretario asistente de Defensa para Asuntos de Seguridad Internacional (2012-2015).
} 
Asociación de Naciones del Sureste Asiático (ASEAN por sus siglas en inglés), entre otras; mientras se apiñan los países de diferentes regímenes políticos que no comparten suficientemente entre sí reglas y normas comunes. La administración Obama intentó extender y dejar arraigadas las normas del «orden internacional liberal» también en el Asia Pacífico.

\subsection{Un legado negativo de Obama}

Al dar un giro en la política exterior de Estados Unidos con relación al Asia, Obama tomó la decisión de no enfrentar un conflicto mayor que podría darse en los años posteriores: la confrontación frente a la China emergente. Como hemos señalado, el objetivo central de la política estadounidense en Asia había sido la de evitar la aparición de un país hegemónico. Ciertamente, antes de la administración de Obama, China no tuvo el poder suficiente para amenazar a la región como país hegemónico y Estados Unidos no se vio en la necesidad de adoptar medidas contra ella. Pero la emergencia de China obligó a Obama a tomar una posición al respecto, optando el presidente por dar mayor importancia a la armonía y la conciliación con China, que a la competencia y la confrontación.

Esta actitud «tolerante» de Obama frente a China se debió a seis factores. Primero, la administración Obama se encontró dominada por una visión optimista o idealista de que China al final aceptaría y compartiría su ideal del «orden internacional liberal», y que actuaría como "parte interesada responsable» de dicho orden.

Segundo, Obama pensó que Estados Unidos necesitaba contar con el apoyo de China para impulsar las agendas importantes de su política exterior. El cambio climático, la no proliferación de armas nucleares, la estabilización de Afganistán, la administración de la economía global, las negociaciones de normas internacionales sobre la seguridad cibernética, entre otras, fueron los temas que Obama pretendió promover, y en los que deseaba contar con la colaboración de China.

Tercero, Obama deseó evitar los efectos negativos de eventuales medidas de represalia económica. China podía tomar medidas de represalia económica en contra de algunas medidas estadounidenses para contener al país asiático. Por ello evitó reaccionar ante determinadas medidas del gigante asiático. Así, en septiembre de 2010, en la zona marítima de las islas de Senkaku —que según China, Japón posee de manera ilegítima - sucedió un choque entre unos barcos pesqueros chinos y un buque patrullero de la guardia costera de Japón; luego del incidente, China prohibió la exportación de tierras raras a Japón ${ }^{6}$.

6 Las tierras raras son empleadas por diversas industrias japonesas; por ello, Japón importó de China casi la tota-
lidad de estas para su uso industrial. Aunque con el paso de tiempo, Japón se recuperó del daño provocado por tal 
Cuarto, Obama se preocupó de no abandonar a los países del Sudeste Asiático que mantenían una colaboración militar-estratégica con Estados Unidos. Los países del Sudeste Asiático buscaban aprovechar la potencial competitividad entre Estados Unidos y China, fortaleciendo las relaciones económicas con el segundo, y contando con el apoyo de Estados Unidos para su seguridad nacional en un eventual caso de emergencia militar. En caso que Estados Unidos confrontase a la potencia asiática cara a cara, China obligaría a cada uno de los países del Sudeste Asiático a elegir entre este y Estados Unidos, amenazando con una represalia económica. Los países medianos y pequeños del Sudeste Asiático optarían por evitar la presión económica de China y desistir de fortalecer las relaciones colaborativas con Estados Unidos en el aspecto militar-estratégico. Por ello, la administración Obama evitó confrontar directamente con China, buscando con ello incrementar el número de países aliados en Asia.

Quinto, China ha ejecutado una serie de actos que cambian el statu quo territorial en Asia a través «agentes paramilitares» como fuerzas de seguridad marítima y milicia marítima; por lo cual, Estados Unidos tampoco puede acudir a las fuerzas armadas.

Sexto, no hubo consenso dentro de la administración respecto de las medidas a ser adoptadas contra China. En la Casa Blanca y la Secretaría de Comercio predominó la opinión de que dichas medidas no cambiarían la posición y actitud de China y solo empeorarían sus relaciones con Estados Unidos; mientras en las secretarías de Estado y Defensa prevaleció la idea de que debía transmitirse una señal a China y a otros países de la región respecto de la firme disposición de Estados Unidos para defender sus intereses en Asia, aunque esto significara sacrificar parte de las buenas relaciones con China.

\subsection{Cambio del statu quo territorial por parte de China}

Finalmente, lo que debemos resaltar es que la posición "tolerante» de Obama no impidió una serie de actos de China para cambiar el statu quo territorial en el Asia, particularmente en el mar de la China Meridional. En cuanto a las armas convencionales, la administración Obama fortaleció la presencia militar en Asia Pacífico, mientras China también incrementó y modernizó sus armas. Ello explica porque China no cambió el statu quo territorial en Asia acudiendo a sus fuerzas armadas?

Sin embargo, China ha venido ejecutando una serie de actos "paramilitares» para cambiar el statu quo territorial en Asia sobre la base de la táctica hábil de acumular paulatinamente hechos consumados. Hasta la actualidad, China ha construido y ocupado un conjunto de islas artificiales por lo menos en siete puntos en el mar

prohibición, mediante la sustitución de las tierras raras y la búsqueda de otros países exportadores de las mismas, en los primeros meses de la represalia china, los efectos negativos para el Japón alcanzaron niveles de consideración.

7 La descripción de los sucesos en Asia se basa en NIDS (2000-2017). 
de la China Meridional que, según su opinión, le pertenecen. En 2016, la Corte Permanente de Arbitraje dictó una sentencia en contra de China respecto de la construcción de estas islas artificiales, sin embargo, el gobierno de Beijing ha hecho caso omiso a esta sentencia y a las críticas internacionales. Asimismo, en el mar de la China Oriental, China ha pretendido impulsar de manera forzosa la exploración de petróleo en la zona marítima sobre la cual unilateralmente reclama soberanía, y ha repetido casi diariamente la invasión a la zona marítima del Japón, como sucedió en el mencionado caso de las islas de Senkaku.

Con relación al mar de la China Oriental, la administración Obama declaró públicamente desde 2010, y en repetidas oportunidades, que las islas de Senkaku constituirían objeto de defensa de acuerdo a lo estipulado en el Tratado de Cooperación Mutua y Seguridad entre Estados Unidos y Japón; no obstante todavía no se ha observado ningún hecho consumado. Pero, en el mar de la China Meridional, China ha avanzado bastante con su táctica de hechos consumados. Recién en octubre de 2015, en la etapa final de su mandato, Obama autorizó la llamada operación «navegación libre» para controlar y disuadir a China de avanzar con la construcción de islas artificiales en la zona.

Pero son pocos los ejemplos de las medidas «directas» que la administración Obama dictó contra la intención de China de cambiar el statu quo territorial en Asia. En la gran mayoría de casos, frente a la táctica china de acumular hechos consumados, Obama optó por tomar medidas «indirectas» como reclamar una solución pacífica de los conflictos, criticar los actos que perjudicaban el control efectivo de otro país, y proponer la congelación de conflictos territoriales. No impulsó activamente medidas «directas» como intimidar, contener y disuadir a través de represalias. Cuando China realizó los actos que intentaban cambiar el statu quo territorial, Obama se limitó a ofrecer a los países aliados y socios ayuda para fortalecer sus fuerzas armadas mediante: ejercicios militares conjuntos, entrenamiento, exportación de armas y dotación de municiones para las fuerzas del orden de estos países. En otras palabras, Estados Unidos no dio la cara para enfrentar a China; los países de la zona debían encarar solos la amenaza de China. De este modo, desde el comienzo hasta el fin de su mandato, Obama mantuvo, en términos generales, la posición de armonía y conciliación, evitando tomar medidas «directas» y elevar la tensión con China.

En lo que concierne a Corea del Norte que amenaza a los países vecinos con las pruebas nucleares y de proyectiles, Obama negó un diálogo directo con el régimen de Pyongyang, y, al mismo tiempo, evitó optar por una intervención militar. En este caso, prácticamente depositó la expectativa en China para presionar al gobierno norcoreano. Si bien es cierto que China — cada vez más irritada por el rebelde régimen de Corea del Norte respecto del desarrollo de las armas nucleares- colaboró con 
Estados Unidos para que las Naciones Unidas impusieran ciertas sanciones contra Corea del Norte, en la etapa final del mandato de Obama, dejó de hacerlo debido a la decisión estadounidense de instalar un nuevo sistema de defensa llamado THAAD ${ }^{8}$ en Corea del Sur.

\section{La política de Trump en Asia}

A más de un año de su toma de posesión, la administración de Trump no ha demostrado una línea diplomático-estratégica clara respecto de Asia. Aparentemente, ha dirigido su interés hacia los asuntos internos, pues no posee una postura firme para tratar las agendas diplomático-estratégicas de modo sistemático y coherente. Más bien, atiende cada agenda diplomático-estratégica sobre la marcha, por asunto o por país. Como hizo público en su campaña electoral, trata las agendas diplomáticoestratégicas como si fueran asuntos de «negocios» o "transacciones» (según Trump, la diplomacia y la política internacional se pueden manejar acudiendo al deal (negociación) con miras a conseguir su objetivo «Primero Estados Unidos»).

En el primer año de su mandato, Trump no puso en práctica casi ninguno de los dislates lanzados durante su campańa electoral como prescindir de los compromisos de defensa con los países aliados y de los tratados estratégicos como el de la OTAN, o la necesidad de dictar medidas proteccionistas contra los productos extranjeros. Pero ha dejado en claro que no tiene ningún interés por mantener y promover el «orden internacional liberal» que Estados Unidos abanderaba hasta la administración antecesora de Obama. Y en el segundo año de su mandato, ha anunciado nuevas iniciativas que profundizan la incertidumbre, confusión y contradicción de su política en Asia.

Para la administración Trump en su primer año de mandato, el asunto de Corea del Norte - vale decir su intención de poseer armas nucleares y misiles balísticos con alcance a Estados Unidos - fue la mayor preocupación entre las agendas diplomático-estratégicas en Asia. De hecho, Trump dedicó más tiempo a dicha cuestión en la parte referente a los asuntos diplomático-estratégicos de su primer Discurso del Estado de la Unión en enero de 2018. Amenazados no solo por las pruebas nucleares con objetivos militares sino también por las pruebas de misiles balísticos que el régimen de Pyongyang empezó en $2017^{9}$, Estados Unidos pretendió disuadir a Corea del Norte de poseer los misiles balísticos con cabeza nuclear mediante las sanciones

\footnotetext{
8 El THAAD es la sigla en inglés de Terminal High Altitude Area Defense (Defensa Aérea de Altitud Alta Terminal). Se trata de un sistema de defensa para derribar misiles balísticos de alcance corto y medio en su trayectoria.

9 Después de retirarse del Tratado de No Proliferación Nuclear en 2003, Corea del Norte ejecutó la primera prueba nuclear en 2006; la segunda en 2009; la tercera en 2013; la cuarta en enero de 2016 (la prueba de termonuclear, según Corea del Norte); la quinta en septiembre de 2016; y la sexta en septiembre de 2017. Pyongyang también hizo pruebas de misiles balísticos en febrero, marzo, abril, mayo, julio, agosto, septiembre y noviembre de 2017.
} 
internacionales por medio de las Naciones Unidas y también por las presiones de China. Asimismo, en su Discurso del Estado de la Unión enfatizó que su gobierno continuaría ejerciendo la mayor presión posible al régimen norcoreano para abandonar su meta de poseer los misiles balísticos con cabeza nuclear.

Sin embargo, a principios de marzo de 2018, se dio a conocer que para mayo se llevaría a cabo una reunión cumbre entre Estados Unidos y Corea del Norte, la que finalmente se concretó en junio. El repentino anuncio de una reunión cara a cara entre Trump y Kim Jong-un — línea diferente o contraria a la que el gobierno de Trump había anunciado- sorprendió a todo el mundo ${ }^{10}$. Resulta impresionante la facilidad o agilidad que posee Trump para cambiar las líneas de conducta, según lo que le parezca con miras a su lema «Primero Estados Unidos». Para los países colindantes con Corea del Norte, la pesadilla sería un escenario en el que Trump permitiera la posesión de armas nucleares y misiles balísticos de corto y mediano alcance por parte de Corea del Norte con la condición de que el régimen de Kim abandone la intención de desarrollar misiles balísticos para alcanzar el territorio de Estados Unidos.

En cuanto a China — que en su primer Discurso del Estado de la Unión Trump mencionó junto con Rusia como «rivales» de Estados Unidos- el primer año del mandato de Trump ha sido más bien un proceso de retorno a la «normalidad» de las relaciones entre las dos potencias. Debe recordarse que, después del triunfo electoral, Trump cuestionó en una entrevista con un medio de comunicación el por qué debía Estados Unidos amarrarse al principio de «una sola China». Esta pregunta aumentó la tensión con la nación asiática, en tanto podía suponer la revocación del llamado "Comunicado de Shanghái», dado a conocer al concluir la visita relámpago del entonces presidente estadounidense Richard Nixon a China en 1972 y que fue parte crucial para la normalización de las relaciones de Estado entre Estados Unidos y China.

En este comunicado, el punto más delicado guardaba relación con Taiwán, porque hasta entonces Estados Unidos había reconocido a Taiwán como la única China, mientras la China comunista seguía afirmando que la isla de Formosa formaba parte de su Estado. Para establecer las relaciones diplomáticas entre Washington y Beijing, ambos países se pusieron de acuerdo en «congelar» la discusión respecto de cuál de las dos, la China comunista o Taiwán, tenía la legitimidad de absorber al otro para conformar una China; por ello, dicho comunicado solo señala que «Estados Unidos se da por enterado (acknowledges) que todos los chinos de ambos lados del Estrecho

\footnotetext{
10 Debemos apuntar que ciertos analistas sobre Corea del Norte ya advertían en la segunda mitad de 2017 que, en las pruebas de misiles balísticos, el Gobierno de Kim evitaba lanzar sus proyectiles hacia la zona de Guam, aunque el régimen de Pyongyang había insistido en incluir dicha zona en sus metas de lanzamiento. Guam es una isla estadounidense, la más cercana de Asia del Este, y se ubica a 3400 kilómetros de Corea del Norte.
} 
de Taiwán sostienen que solo hay una China y que Taiwán es parte de China. El gobierno de Estados Unidos no desafía esa posición. Reafirma su interés en una solución pacífica de la cuestión de Taiwán por los propios chinos».

En febrero de 2017, Xi Jinping, presidente de la República Popular China, accedió a la primera conversación telefónica con Trump, luego de la cual la Casa Blanca dio a conocer un comunicado donde señalaba que «el presidente Trump accedió a respetar nuestra política de una China, a pedido del presidente Xi Jinping». De este modo, ambos países zanjaron el primer desencuentro y abrieron paso a la primera cumbre entre Trump y Xi en abril de 2017 en Estados Unidos; y la segunda en noviembre del mismo año en China.

Mientras tanto, la administración Trump continuó con la operación «navegación libre» de Obama, y la reanudó en mayo de 2017. Hasta enero de 2018, ejecutó dicha operación cinco veces ${ }^{11}$. Aunque Estados Unidos — acudiendo al paso inocente consagrado por la Convención de las Naciones Unidas sobre el Derecho del Mar de 1982 - se cuida de no anclar sus buques en las aguas territoriales proclamadas por China, el gobierno de Beijing se ha mostrado cada vez más irritado frente al acto de demostración de fuerza por parte de su rival estadounidense.

Las relaciones entre Estados Unidos y China amenazaron con empeorarse con el nuevo anuncio de Trump respecto de las importaciones de China en marzo de 2018. A principios de ese mes, Trump declaró la imposición de aranceles a los productos de fierro y aluminio de China y, a fines del mismo, anunció la imposición de aranceles a China por un valor de hasta 60000 millones de dólares anuales por supuestas afrentas a la propiedad intelectual estadounidenses. China ha reaccionado de manera enérgica particularmente al segundo anuncio.

En lo concerniente al comercio internacional, inmediatamente después de la toma de posesión, Trump anunció el retiro de Estados Unidos del Acuerdo Transpacífico de Cooperación Económica (TPP por sus siglas en inglés). En enero de 2018, el presidente estadounidense se refirió a la posibilidad del retorno de Estados Unidos al acuerdo pero impuso como condición la renegociación de dicho acuerdo; sin embargo, en su primer Discurso del Estado de la Unión no tocó el tema.

\section{Conclusiones: la política estadounidense en Asia a la deriva}

En el siglo XX, Estados Unidos tuvo la política diplomático-estratégica de comprometerse con el «orden internacional liberal» y, al igual que en Europa Occidental,

11 La operación fue realizada en mayo, julio, agosto, octubre de 2017 y en enero de 2018. 
evitar la aparición de un país hegemónico en Asia. Dicha política se basaba en el consenso bipartidario entre los agentes políticos de Washington. Aunque la superpotencia se vio obligada a intervenir en la zona dos veces, en la guerra de Corea y la de Vietnam, con el masivo despliegue de sus tropas, siempre le dio prioridad a Europa Occidental, y al finalizar la intervención militar en Asia, su mayor interés retornó inmediatamente al otro lado del océano Atlántico, donde hasta la década de 1970 se encontraba el centro de gravedad de las actividades del ser humano, principalmente las económico-comerciales. Dicho centro de gravedad se trasladó del océano Atlántico al Pacífico en las décadas de 1980 y 1990, y en este siglo, en la administración Obama, Estados Unidos decidió dar un giro a la política diplomático-estratégica y otorgar mayor importancia al Asia.

Aunque la decisión de Obama correspondió al cambio mundial de la dinámica de las actividades del ser humano, dejó un legado negativo para el futuro de Asia: con su actitud «tolerante» - basada en una esperanza optimista e idealista de que China aceptaría y compartiría el ideal estadounidense del «orden internacional liberal», en otras consideraciones diplomático-económicas, así como en la situación económica interna del país-, se abstuvo de demostrar la posición de confrontación frente a la China emergente. Dio prioridad a la armonía y conciliación —sobre la competencia y la confrontación - con China. Mientras Obama mantuvo dicha posición en su mandato, China se dedicó a implementar una serie de acciones para cambiar el statu quo territorial en Asia, particularmente en el mar de la China Meridional. Sobre la base de la táctica de acumular paulatinamente hechos consumados, y contra la opinión pública internacional y la sentencia de la Corte Permanente de Arbitraje, el país asiático ha construido islas artificiales por lo menos en siete puntos en dicho mar que, según su opinión, le pertenecen.

Con la llegada de Trump a la presidencia — que simboliza el retorno al aislacionismo de Estados Unidos—, la situación se ha puesto más confusa e contradictoria y todavía hay muchas cosas por definir. Sin embargo, la administración de Trump ha dedicado una mayor atención, entre las agendas diplomático-estratégicas en el primer año de su mandato, a los asuntos que involucran a Corea del Norte. El presidente estadounidense ha optado repentinamente por la línea del diálogo, luego de continuar con la política de presión y sanción como sus antecesores. Con China, país rival junto con Rusia, según su última evaluación, Trump retornó a las relaciones «normales» en su primer año de su mandato, pero al empezar el segundo año, declaró la "guerra comercial» contra el gigante asiático. Aunque ha quedado claro que Trump no se interesa por mantener y promover el «orden internacional liberal» que la superpotencia persiguió en el siglo pasado, todavía no se sabe exactamente qué dirección o rumbo va a tomar su administración con relación a cada uno de 
los asuntos concretos de la agenda político-estratégica de las relaciones con Asia; tampoco existen elementos suficientes para establecer un juicio sobre en qué grado o hasta dónde Estados Unidos se retirará de Asia ${ }^{12}$.

El cambio de la línea diplomático-estratégica en Asia emprendida por Obama no se arraigó. El sucesor Trump no ha demostrado una línea clara respecto de sus relaciones con Asia hasta el momento. Con Trump, la política estadounidense en Asia ha quedado a la deriva, aumentando la incertidumbre y la volatilidad por su carácter "populista».

\section{Referencias}

Anderson, N.D. y Cha, V.D. (2017). The Case of the Pivot to Asia: System Effects and the Origins of Strategy. Political Science Quarterly, 132(4), 595-617. https://doi. org/10.1002/polq. 12703

Bader, J. (2012). Obama and China's Rise: An Insider's Account of America's Asia Strategy. Washington, D.C.: Brookings Institution Press.

Campbell, K.M. (2016). The Pivot: The Future of American Statecraft in Asia. Nueva York: Grand Central Publishing.

Chollet, D. (2016). The Long Game: How Obama Defied Washington and Redefined America's Role in the World. Nueva York: Public Affairs.

Clinton, H. R. (2011). America's Pacific Century. Foreign Policy, 189, 56-63.

Clinton, H.R. (2014). Hard Choices. Nueva York: Simon \& Schuster.

Gaddis, J.L. (1987). The Long Peace: Inquiries into the History of the Cold War. Nueva York: Oxford University Press.

Gaddis, J.L. (2005a). The Cold War: A New History. Nueva York: The Penguin Press.

Gaddis, J.L. (2005b). Strategies of Containment: A Critical Appraisal of Postwar American National Security Policy. Nueva York: Oxford University Press.

Goh, E. (2015). The Struggle for Order: Hegemony, Hierarchy and Transition in Post-Cold War Asia. Oxford: Oxford University Press.

Kissinger, H. (1994). Diplomacy. Nueva York: Simon \& Schuster.

Kissinger, H. (2014). World Order. Nueva York: The Penguin Press.

Leffler, M.P. (2005). National Security and US Foreign Policy. En M.P. Leffler y D.S. Painter (Eds.), Origins of the Cold War: An International History (pp. 15-41). 2da. ed. Nueva York: Routledge.

Levitsky, S. y Ziblatt, D. (2018). How Democracies Die: What History Reveals About Our Future. Nueva York: Viking.

\footnotetext{
12 Podemos agregar otra piedra de toque para la futura línea diplomático-estratégica de Estados Unidos: si retira o no sus bases militares de Corea del Sur. Ya a principios de esta década, en la súper potencia existe esta opinión; inclusive, ha sido propuesta por parte de militares y analistas conservadores.
} 
Mecanic, J. (2017). Donald Trump, The Accidental President: This Was Never about the Job-It Was about Fame. HuffPost. Recuperado de https://www.huffingtonpost.com/ entry/the-accidental-president_us_591f4d0ee4b07617ae4cbbda

Murakami, Y. (2012). Asia del Este y la política exterior del Japón: desafíos para el siglo XXI. Agenda Internacional, 29, 19-54.

Murakami, Y. (2013). Neoliberalismo, Sistema de partidos políticos y 'giro a la izquierda': dinámica política en la América Latina posneoliberal. En Y. Murakami (Ed.), América Latina en la era posneoliberal: democracia, conflictos y desigualdad (pp. 9-61). Lima: Instituto de Estudios Peruanos.

Murakami, Y. (2017). Dinámica cambiante en las relaciones internacionales e interamericanas antes y después del cambio de siglo. En Y. Murakami (Ed.), Desarrollo, integración y cooperación en América Latina y Asia-Pacifico: perspectivas y rol de Japón (pp. 9-32). Lima: Instituto de Estudios Peruanos.

National Institute for Defense Studies (NIDS). (2000-2017). East Asian Strategic Review 2000-2017. Tokio: NIDS. (Publicación anual del NIDS afiliado al Ministerio de Defensa del Japón sobre el tema).

Silove, N. (2016). The Pivot before the Pivot: U.S. Strategy to Preserve the Power Balance in Asia. International Security, 40(4), 45-88. https://doi.org/10.1162/ISEC_a_00238

U.S. Departament of Defense. (1995). East Asia Strategy Report. NAPSNet Special Reports. Recuperado de https://nautilus.org/napsnet/napsnet-special-reports/ east-asia-strategy-report/

Fecha de recepción: 16 de marzo de 2018

Fecha de aceptación: 4 de mayo de 2018 